\title{
LncRNA NORAD/miR-202-5p regulates the drug resistance of A549/DDP to cisplatin by targeting P-gp
}

\author{
Jian-Guo Shen ${ }^{1}$, Shi-Ning $\mathrm{Xu}^{2}$ and Li-Guo Yin ${ }^{3}$ \\ ${ }^{1}$ Department of Emergency Medicine, Jinan Central Hospital, Jinan, Shandong, P.R. China \\ ${ }^{2}$ Department of Thoracic Surgery, Qianfoshan Hospital, Jinan, Shandong, P.R. China \\ ${ }^{3}$ Department of Thoracic Surgery, Jinan Central Hospital, Jinan, Shandong, P.R. China
}

\begin{abstract}
In this study, we established the DDP-resistant NSCLC cell line A549/DDP to detect the effect of NORAD on cisplatin resistance of A549/DDP cells. NORAD was highly expressed in A549/DDP cells compared with A549 cells. The MTT data showed that knockdown of NORAD enhanced the inhibition rate of cisplatin on the A549/DDP cells and decreased IC50 value. The colony formation and MTT assay suggested that cisplatin inhibited cell proliferation, and knockdown of NORAD enhanced the inhibitory effect of cisplatin on A549/DDP cells. Besides, we found that NORAD silence reduced the P-gp expression but not BCRP, LRP and MRP. Moreover, NORAD could directly bind to miR-202-5p, and ABCB1 was a target of miR-202-5p. The MTT assay found that miR-202-5p inhibitor reversed the effects of NORAD silence on cisplatin resistance of A549/DDP cells. Then, the Western blot data showed that knockdown of NORAD reduced P-gp expression, and miR-202-5p inhibitor enhanced P-gp expression. ABCB1 overexpression reversed the inhibitory effect of NORAD knockdown on A549/DDP cells. Moreover, NORAD could directly bind to miR-202-5p, and ABCB1 was a target of miR-202-5p. Inhibition of miR-202-5p and overexpression of $\mathrm{ABCB} 1$ eliminated the effects of NORAD silence on cisplatin resistance of A549/DDP cells. Overexpression of miR-202-5p suppressed P-gp expression in A549/DDP cells. Collectively, our data showed that NORAD could enhance the DDP resistance of A549/DDP cells and potentially increased P-gp expression by sponging the miR-202-5p.
\end{abstract}

Key words: NORAD - miR-202-5p - A549/DDP - P-gp

\begin{abstract}
Abbreviations: ABCB1, ATP-binding cassette subfamily B member 1; BRCP, breast cancer resistance protein; $\mathrm{CDNA}$, complementary deoxyribonucleic acid; ceRNA, competing endogenous RNAs; CRC, colorectal carcinoma; DDP, cisplatin; FCS, foetal calf serum; Has-miR-202-5p, homo sapiens-miRNA 202-5p; LRP, lung resistance protein; MDR 1, multidrug resistance protein 1; MRP, multidrug resistance-associated protein; MTT, 3-(4,5)-dimethylthiahiazo(-z-y1)-3,5-di-phenytetrazoliumromide; NORAD, noncoding RNA activated by DNA damage; NSCLC, non-small cell lung cancer; OD, optical density; P-gp, permeability glycoprotein; qRT-PCR, quantitative real-time polymerase chain reaction; RIP, RNA immunoprecipitation; SDS, sodium dodecyl sulphate.
\end{abstract}

\section{Introduction}

Lung cancer is histologically divided into two main types: small cell lung cancer and non-small cell lung cancer

Correspondence to: Li-Guo Yin, Jinan Central Hospital, 105 Jiefang Road, Jinan, Jinan, Shandong 250013, P.R. China E-mail: yingliguo123@163.com
(NSCLC). NSCLC is one of the most common and fatal malignant tumours worldwide (Barton 2017). For patients who are diagnosed with unresectable tumours at advanced stage, systemic chemotherapy is an irreplaceable treatment. Cisplatin (DDP) is a first-line chemotherapeutic agent used to treat advanced NSCLC in the past 20 years (Arbour et al. 2019). However, considering that some patients experience drug resistance to DDP, it often fails clinically (Chen et al. 
2018). Therefore, drug resistance has become a major challenge in the treatment of NSCLC, and underlying molecular mechanisms of drug resistance to DPP are not yet well understood.

Disorder of long noncoding ribonucleic acids (lncRNAs) has been observed in several human diseases and has contributed to disease progression (Schmitt et al. 2016; Rafiee et al. 2018). Previous studies have shown that lncRNAs are associated with the development, metastasis, and diagnosis of NSCLC (Lu et al. 2018). It has been reported that some lncRNAs contribute to DDP resistance in NSCLC (Hu et al. 2017; Tian et al. 2019). Recently, noncoding RNA activated by DNA damage (NORAD) has attracted attention because of its promoting role in tumour progression (Liu et al. 2016; Li et al. 2018a; Zhang et al. 2019). Zhang et al. have shown that NORAD promotes colorectal tumour growth by inhibiting miR-202-5p (Zzhang et al. 2018). Moreover, IncRNA NORAD is also reported to be capable of accelerating the chemoresistance of NSCLC cells by targeting miR-129-1-3p (Huang et al. 2020). However, whether NORAD regulates the proliferation and resistance of A549/DDP cells remains unclear, and its underlying mechanism has not been revealed.

ATP-binding cassette sub-family B member 1 (ABCB1), also known as permeability glycoprotein (P-gp) or multidrug resistance protein 1 (MDR 1), is an important member of the ABC transporter family (Aller et al. 2009; Wu et al. 2011). P-pg is an important cell membrane protein that pumps several foreign substances out of the cells. P-gp, as a drug pump, can pump DDP out of the cancer cells. Thus, the drug concentration in cells is reduced. Moreover, the down-regulation of $\mathrm{ABCB} 1$ enhances the cytotoxic effects of DDP in ovarian cancer ( $\mathrm{Li}$ et al. 2018b). However, whether lncRNA NORAD could mediate drug resistance in DDPresistant NSCLC cells by regulating ABCB1 remains unclear.

This study investigated the effect of NORAD on the DDP resistance NSCLC cell line A549/DDP and its potential mechanism. Our results showed that NORAD is highly expressed in NSCLC cell A549 with resistance to DDP (A549/ DDP). Silencing NORAD inhibited DDP resistance and P-gp expression in A549/DDP cells. Further studies demonstrated that NORAD could directly bind to miR-202-5p, and the reduced resistance induced by silencing NORAD in A549/ DDP was reversed by miR-202-5p inhibitor by promoting P-gp expression. Our findings may provide an additional insight into the role of NORAD in the progression of NSCLC.

\section{Materials and Methods}

\section{Cell culture}

The DDP-resistant NSCLC cell line A549/DDP was established based on Yan et al.'s description (Chian et al. 2019).
The progenitor A549 cells were first treated with a dose of DDP $(0.2 \mu \mathrm{g} / \mathrm{ml})$. Subsequently, the cells were treated with increasing concentrations of DDP at a range of 0.05 to $1.0 \mu \mathrm{g} / \mathrm{ml}$ in RPMI-1640 medium with $10 \%$ foetal calf serum (FCS) in a $37^{\circ} \mathrm{C}$ humidified incubator supplied with $5 \% \mathrm{CO}_{2}$. Then, the selected cells that demonstrated resistance to DDP (A549/DDP) and A549/DDP cells were regularly maintained in complete RPMI-1640 medium supplemented with DDP $(0.05 \mu \mathrm{g} / \mathrm{ml})$ in a $37^{\circ} \mathrm{C}$ humidified incubator supplied with $5 \% \mathrm{CO}_{2}$. The cell morphologies of A549 and A549/DDP cells were observed using a phase-contrast microscope (Nikon, Tokyo, Japan).

\section{Cell transfection}

NORAD siRNA and its corresponding negative control (NC) (Sangon Biotech, Shanghai, China) were transfected into A549/DDP cells for $48 \mathrm{~h}$ for loss-of-function experiments using Lipofectamine 2000 (Invitrogen, CA, USA) according to the manufacturer's instructions. miR-202-5p mimics, miR-202-5p inhibitor, and their vector (NC) and ABCB1 overexpression plasmid (pcDNA3.1 ABCB1, pc$\mathrm{ABCB} 1$ ) and its corresponding NC (RiboBio, Guangzhou, China) were, respectively, co-transfected into A549/DDP cells with NORAD siRNA. After a 6-h transfection, the medium was replaced with RPMI-1640 medium containing 10\% FCS. The untreated cells served as blank control group (Mock group).

\section{Quantitative real-time polymerase chain reaction ( $q R T-P C R$ )}

Total RNA was obtained from the cells using a TRIzol reagent (Invitrogen, CA, USA) according to the manufacturer's instructions. Subsequently, mRNA was reverse-transcribed into cDNA using a First-strand cDNA synthesis kit (OriGene Technologies, MD, USA). Subsequently, the relative expression was detected by the ABI7500 PCR system (ABI) using $\mathrm{SYBR}^{\oplus}$ Green Master Mix (Takara). The conditions for RTPCR reaction were as follows: initial denaturation at $95^{\circ} \mathrm{C}$ for $5 \mathrm{~min}$, followed by $40 \mathrm{cycles}$ of $95^{\circ} \mathrm{C}$ for $10 \mathrm{~s}, 60^{\circ} \mathrm{C}$ for $20 \mathrm{~s}$, and $72^{\circ} \mathrm{C}$ for $34 \mathrm{~s}$. The relative expression was calculated using the $2^{-\Delta \Delta C t}$ method. The qPCR primers were as follows: NORAD (forward 5'-AAGCTGCTCTCAACTCCACC-3', reverse 5'-GGACGTATCGCTTCCAGAGG-3'), miR-202-5p (forward 5'-ACACTCCAGCTGGGTTTCTTCATATACGT-3', reverse 5'-TGGTGTCGTGGAGTCG-3'), ABCB1 (forward 5'-GAACCTGTATTGTTTGCCACC-3', reverse 5'-ACTCCATCATCGAAACCAGC-3'), breast cancer resistance protein (BCRP) (forward 5'-CGGGATCCATGTCTTCCAGTAATGTCGAAGTT-3', reverse 5'-CCGCTCGAGTTAAGAATATTTTTTAAGAAATAAC-3'), lung resistance protein (LRP) (forward 5'-GACAGTTCACAGTGTTGTCC-3', reverse 5'-GCGTGACGACAGAAACCGAA-3'), multidrug resistance-associated protein (MRP) (forward 5'-CAA- 
GGTGGATGCGAATGAG-3', reverse 5'-GGCCCAAAGGTCTTGTATAAC-3'), and glyceraldehyde-3-phosphate dehydrogenase (GAPDH) (forward 5'-TGCCAAATATGACATCAAGAA-3', reverse 5'-GGAGTGGGTGTCGTCGCTGTTG-3'). GAPDH was used as the reference gene.

\section{Western blot analysis}

The concentration of the protein lysates was detected using BCA kit (Thermo Fisher, MA, USA). All the protein lysates were separated using $10 \%$ sodium dodecyl sulphatepolyacrylamide gel electrophoresis (SDS-PAGE) and transferred onto a polyvinylidene fluoride (PVDF) membrane. Subsequently, the PVDF membrane was blocked with 5\% skimmed milk for $1 \mathrm{~h}$ and incubated with primary antibodies, including GAPDH, P-gp, BCRP, LRP, and MRP (CST, MA, USA, diluted at 1:1000) overnight at $4^{\circ} \mathrm{C}$. Then, after washing in TBST thrice, the membranes were incubated with goat anti-rabbit horseradish peroxidase-conjugated secondary antibodies for $1 \mathrm{~h}$. Finally, freshly made electrochemiluminescent solution was added for coloration and exposed in the dark. The ImageJ software (Bio-Rad, Richmond, CA, USA) was used to analyse the grey values. GAPDH was used as a control.

\section{Dual-luciferase reporter assay}

StarBase (http://starbase.sysu.edu.cn/) was used to predict the regulation of miR-202-5p by NORAD. The wild-type (WT) (NORAD-WT) or mutant (MUT) (NORAD-MUT) fragments of NORAD 3'UTR containing miR-202-5p binding site were synthesised. The WT or MUT sequence was cloned into a pGL3 luciferase control reporter vector (Promega, WI, USA). A549/DDP cells $\left(5 \times 10^{5}\right)$ were seeded in 24-well plates for $24 \mathrm{~h}$. Subsequently, miR-202-5p mimics or mimics NC were transfected into A549/DDP cells, respectively, by Lipofectamine 2000 (Invitrogen, CA, USA). After a 48-h transfection, luciferase activity was measured using the Dual-Luciferase Reporter Assay Kit (Promega, WI, USA).

For the detection of miR-202-5p binding to ABCB1 mRNA by dual-luciferase activity assay, the ABCB1-WT or ABCB1-MUT fragments of ABCB1 3'UTR containing miR-202-5p binding site were synthesised, and WT or MUT sequence was cloned into a pGL3 luciferase control reporting vector. After a 24-h culture in 24-well plates, A549/DDP cells were transfected with miR-202-5p mimics or mimics NC. After a 48-h transfection, luciferase activity was measured.

\section{RNA immunoprecipitation (RIP) assay}

The Magna RIPTM RNA Binding Protein Immunoprecipitation kit (RIP, Millipore, MA, USA) was used to analyse the binding capacity between NORAD and miR-202b-5p. Cell lysate (A549) was preserved in RIP buffer containing magnetic beads precoated by antibodies of Ago2 or immunoglobulin G (IgG). Normal IgG was used as controls. The relative expression levels of NORAD and miR-202b-5p were measured by qRT-PCR.

\section{Colony formation assay}

The A549 or A549/DDP cells were plated into 6-well plates and cultured for 14 days. The colonies were fixed with $10 \%$ formaldehyde for $30 \mathrm{~min}$ and subsequently stained with $0.5 \%$ crystal violet for $5 \mathrm{~min}$. The colonies were photographed and quantified using a camera (Olympus, Tokyo, Japan).

\section{3-(4,5)-Dimethylthiahiazo(-z-y1)-3,5-di-phenytetrazolium- bromide (MTT) assay}

The MTT assay was performed based on Dong et al.'s description (Shah et al. 2020). The transfected cells were resuspended in RPMI-1640 with 10\% FCS and seeded into 96 -well plate $\left(6 \times 10^{3}\right)$, followed by incubation at $37^{\circ} \mathrm{C}$ with $5 \% \mathrm{CO}_{2}$ for 24,48 , and $72 \mathrm{~h}$. Subsequently, $20 \mu \mathrm{l}$ of MTT reagent $(5 \mathrm{mg} / \mathrm{ml}$, Sigma, MO, USA) was added into the cells. After incubation for $4 \mathrm{~h}, 150-\mu \mathrm{l}$ dimethyl sulphoxide was supplemented to each well. The mixture was subsequently shaken at low speed for 10 min to sufficiently dissolve the crystals. The $\mathrm{OD}_{490}$ absorbance values for the samples were measured using the enzyme-linked immunometric metre.

\section{Statistical analyses}

All data are representative of three independent experiments and expressed as mean \pm standard deviation (SD). Differences between the two groups were analysed by T-test, and differences among groups were analysed by analysis of variance test using the Statistical Package for the Social Sciences version 22.0 (Chicago, IL, USA) and GraphPad Prism version 7.01 (version X; La Jolla, CA, USA) software. A value $p<0.05$ was considered statistically significant.

\section{Results}

High expression of NORAD is observed in A549/cisplatin (DDP) cells

The cell morphologies of A549 and A549/DDP cells were observed under a phase-contrast microscope after the successful DDP-resistant NSCLC cell line establishment. Compared with the A549 cells, A549/DDP cells were fusiform and arranged in a spiral or radial shape, similar 
to fibre cells. The inhibition rates of DDP on the A549 cells and A549/DDP cells were analysed by the MTT assay. The results showed that the inhibition rate of DDP on the A549/DDP cells is lower than that on the A549 cells $(p<0.05$, Fig. 1A). Meanwhile, qRT-PCR demonstrated that the expression of NORAD is significantly higher in the A549/DDP cells than that in the A549 cells $(p<0.05$, Fig. 1B).

Silencing NORAD suppresses the cisplatin resistance of A549/DDP cells

To further study the effect of NORAD on DDP resistance in A549/DDP cells, we used siRNA to knockdown NORAD. The results showed that the expression of NORAD in the A549/DDP cells transfected with si-NORAD (the si-NORAD group) is significantly lower than that in the A549/ DDP cells transfected with the control vector (the si-NC group) ( $p<0.05$, Fig. $2 \mathrm{~A}$ ), indicating that RNA interference was successful. The MTT assay showed that NORAD knockdown better increases the inhibition rate of DDP on the A549/DDP cells compared with the si-NC group (Fig. 2B). Data of colony formation assay suggested that the colony numbers of si-NORAD group are lower than those in the si-NC group $(p<0.05$, Fig. $2 \mathrm{C})$. The $\mathrm{IC}_{50}$ value of DDP was lower in the si-NORAD group than that in the si-NC group ( $p<0.05$, Fig. 2D). Moreover, qRT-PCR and Western blot analysis demonstrated that NORAD knockdown decreases the expression of the ABCB1 mRNA and drug resistance protein $\mathrm{P}$-gp in the si-NORAD group compared with si-NC group or Mock group ( $p<0.05$, Fig. $2 \mathrm{E}$ and F). However, there were no evident changes in the expression of drug resistance proteins BCRP, LRP, and MRP in the siNC group and Mock group compared with the si-NORAD group ( $p>0.05$, Fig. $2 \mathrm{~F})$.

\section{NORAD activates as a competing endogenous RNA of miR-202-5p}

StarBase (http://starbase.sysu.edu.cn/) was used to predict the binding site of NORAD to miR-202-5p (Fig. 3A). The
miR-202-5p expression was higher in the si-NORAD group than that in the si-NC group ( $p<0.05$, Fig. $3 B$ ). The NORAD expression also increased after down-regulating miR-202$5 p$ in A549/DDP cells using the miR-202-5p inhibitor $(p<$ 0.05 , Fig. 3C). We conducted luciferase reporter assays after transfecting A549/DDP cells containing synthetic miR202-5p mimics with the WT or MUT miR-202-5p target sequences in NORAD. We found that the miR-202-5p mimic reduced luciferase activity in the cells transfected with the WT sequences but had no effect on luciferase activity in cells transfected with the MUT sequences (Fig. 3D). These data indicated that miR-202-5p may be a target of NORAD, and NORAD might promote DDP resistance by targeting miR-202-5p. The result of anti-Ago2 RIP assay further confirmed that miR-202-5p is the target gene of lncRNA NORAD (Fig. 3D).

miR-202-5p inhibition attenuates the effect of si-NORAD on cisplatin resistance in A549/DDP cells

To investigate whether miR-202-5p is involved in the promotion effect of NORAD on DDP resistance of A549/DDP cells, we co-transfected si-NORAD and miR-202-5p inhibitors into the A549/DDP cells, and the results showed that NORAD knockdown decreases the DDP resistance of A549/ DDP cells, whereas the inhibition of miR-202-5p increases the DDP resistance of A549/DDP cells. Additionally, the inhibition of miR-202-5p eliminated the effect of NORAD knockdown $(p<0.05$, Fig. $4 \mathrm{~A}$ and B).

\section{P-gp is the target of NORAD/miR-202-5p}

Bioinformatics analysis indicated that miR-202-5p can directly bind to 3'-untranslated regions (3'-UTRs) of P-gp (Fig. 5A). Moreover, miR-202-5p mimics significantly decreased P-gp level in A549/DDP cells (Fig. 5B). Based on the predicted binding sites on WT P-gp, we designed the dual-luciferase reporter gene assay. The results showed that miR-202-5p mimics significantly inhibits the relative luciferase activity of WT P-gp ( $p<0.05$, Fig. 5 C), suggesting that miR-202-5p targets ABCB1. The anti-Ago2 RIP
A

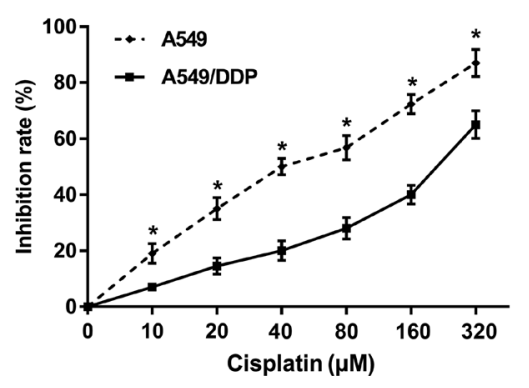

B

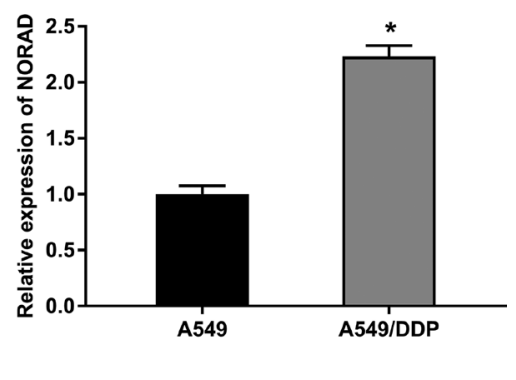

Figure 1. High expression of NORAD in A549/DDP cells. A. The cell inhibition rate of A549/DDP cells and A549 cells detected by the 3-(4,5)-dimethylthiahiazo(-z-y1)-3,5-diphenytetrazoliumromide (MTT) assay. B. The expression of NORAD in A549/DDP cells and A549 cells detected by qRT-PCR. Data are shown as means $\pm \mathrm{SD}$ from three independent experiments. ${ }^{*} p<0.05$ compared with the A549 group. DDP, cisplatin. 


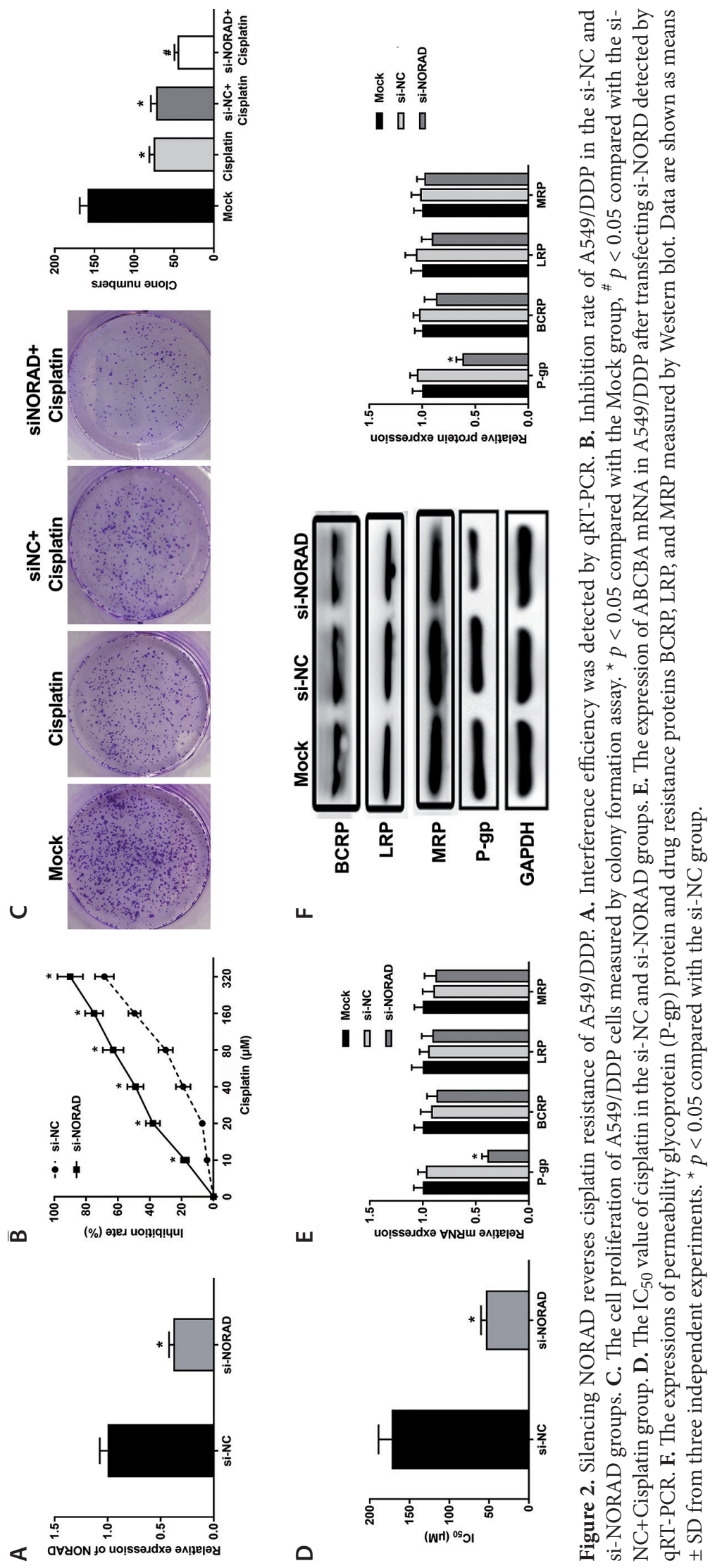


A NORAD UACAAUUCAUAGGAA
hsa-miR-202-5p CGUAUCCUU

\section{B}

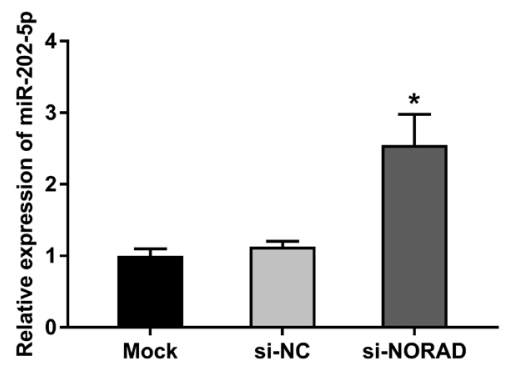

D

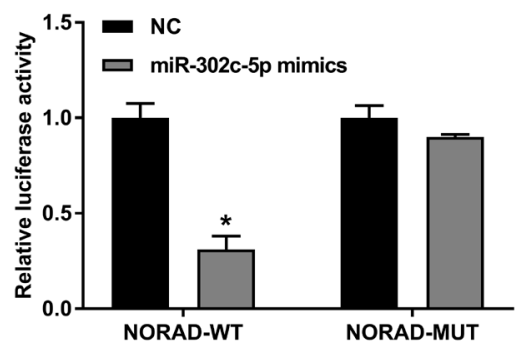

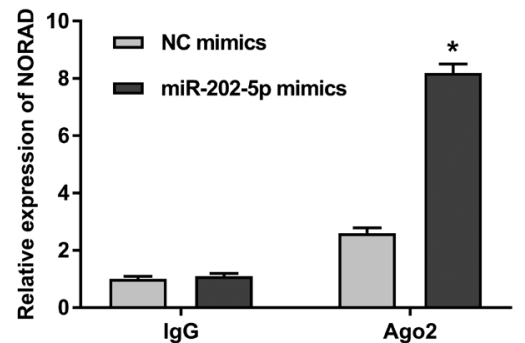

C

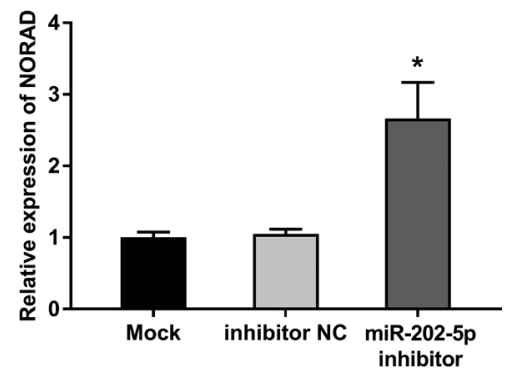

Figure 3. NORAD regulates the expression of miR-202-5p. A. Using StarBase (http://starbase.sysu.edu.cn/) to predict the binding site of NORAD to microRNA-202-5p. B. The expression of miR-202-5p in A549/DDP cells detected by qRT-PCR. C. The expression of NORAD in A549/DDP cells detected by qRT-PCR. D. Validation of NORAD binding to miR-202-5p by dual-luciferase activity assay and anti-Ago2 RIP assay. Data are shown as means \pm SD from three independent experiments. ${ }^{*} p<0.05$ compared with the si-NC, inhibitor NC, or mimics NC group.

assay further confirmed the targeting association between miR-202-5p and ABCB1 (Fig. 5C). qRT-PCR and Western blot analysis results illustrated that the knockdown of miR-202-5p causes a regain of ABCB1 mRNA and P-gp protein levels, which were decreased by NORAD silencing (Fig. 5D).

$A B C B 1$ overexpression weakens the effect of si-NORAD on the cisplatin resistance in A549/DDP cells

The pc-ABCB1 and si-NORAD were co-transfected into the A549/DDP cells to determine whether $A B C B 1$ is associated with the effect of NORAD on DDP resistance in A549/DDP cells. The data suggested that NORAD knockdown decreases the DDP resistance of A549/DDP cells, whereas the overexpression of $\mathrm{ABCB} 1$ eliminates the effect of si-NORAD on DDP resistance of A549/DDP cells $(p<0.05$, Fig. 6).

\section{Discussion}

NSCLC is the most prevalent type of lung cancer, with poor prognosis and high reoccurrence rate among patients (Ramanathan et al. 2019). Chemotherapy is one of the primary options for patients with unresectable tumours. However, the reoccurrence rate of NSCLC is still high considering that some patients with NSCLC are
A

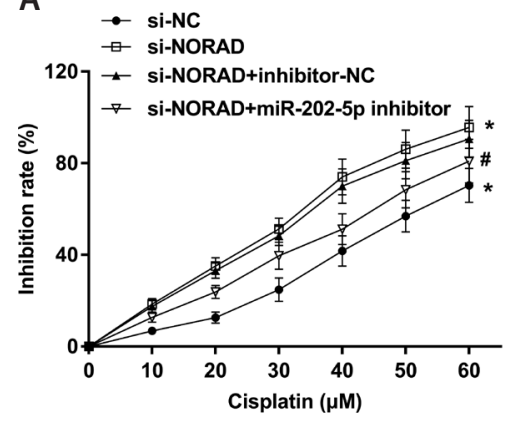

B

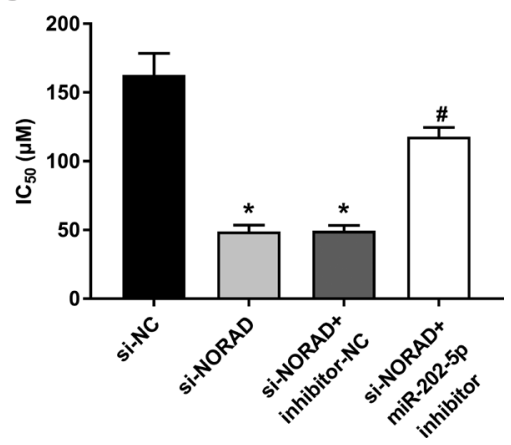

Figure 4. miR-202-5p eliminates the effect of NORAD in A549/DDP cells. A. The inhibition rate in A549/DDP co-transfected with si-NORAD and miR-202-5p inhibitor. B. The $\mathrm{IC}_{50}$ value in A549/DDP co-transfected with si-NORAD and miR-202-5p inhibitor. Data are shown as means \pm SD from three independent experiments. ${ }^{*} p<0.05$ compared with the si-NC group, ${ }^{*} p<0.05$ compared with the siNORAD+inhibitor-NC group. 
chemoresistant to chemotherapy (Nagasaka et al. 2018). It has been reported that IncRNAs participate in multiple cancers and regulate the chemoresistance of cancer cells, including NSCLC (Nagasaka et al. 2018). NORAD has been discovered to be a tumour promoter in several cancers (Tao et al. 2019; Tong et al. 2019), but little is known whether NORAD can affect the drug resistance of NSCLC cells. In the present study, we investigated the role of NORAD in drug resistance of A549/DDP cells and its potential mechanism. We showed that NORAD is more up-regulated in the A549/DDP cells compared with the A549 cells. By silencing NORAD, we demonstrated that

\section{A ABCB1 3'UTR GGAAAAAAAAAUAGGAAA \\ miR-202-5p UUCAUAUACGUAUCCUUU}

B

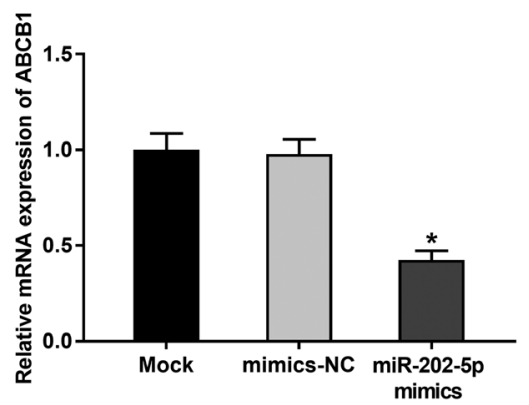

C

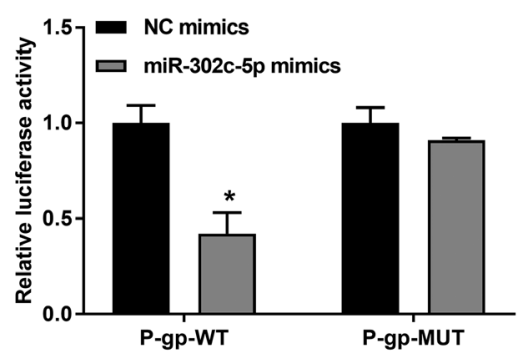

D

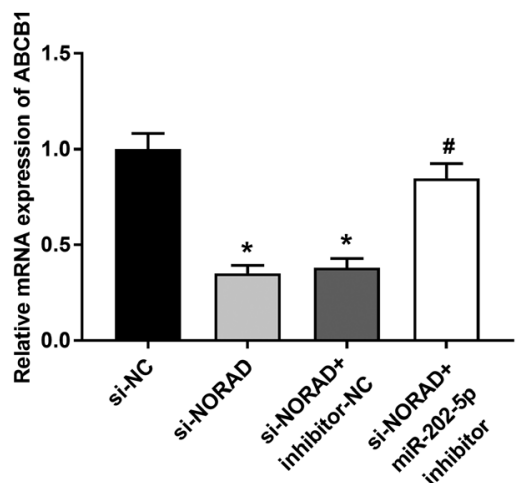

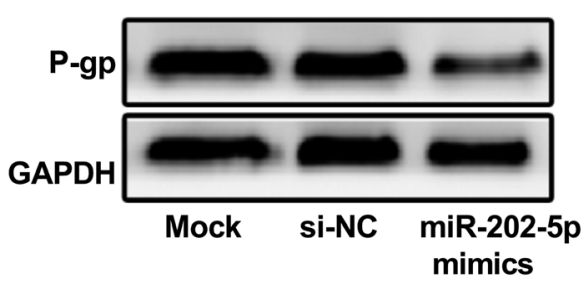
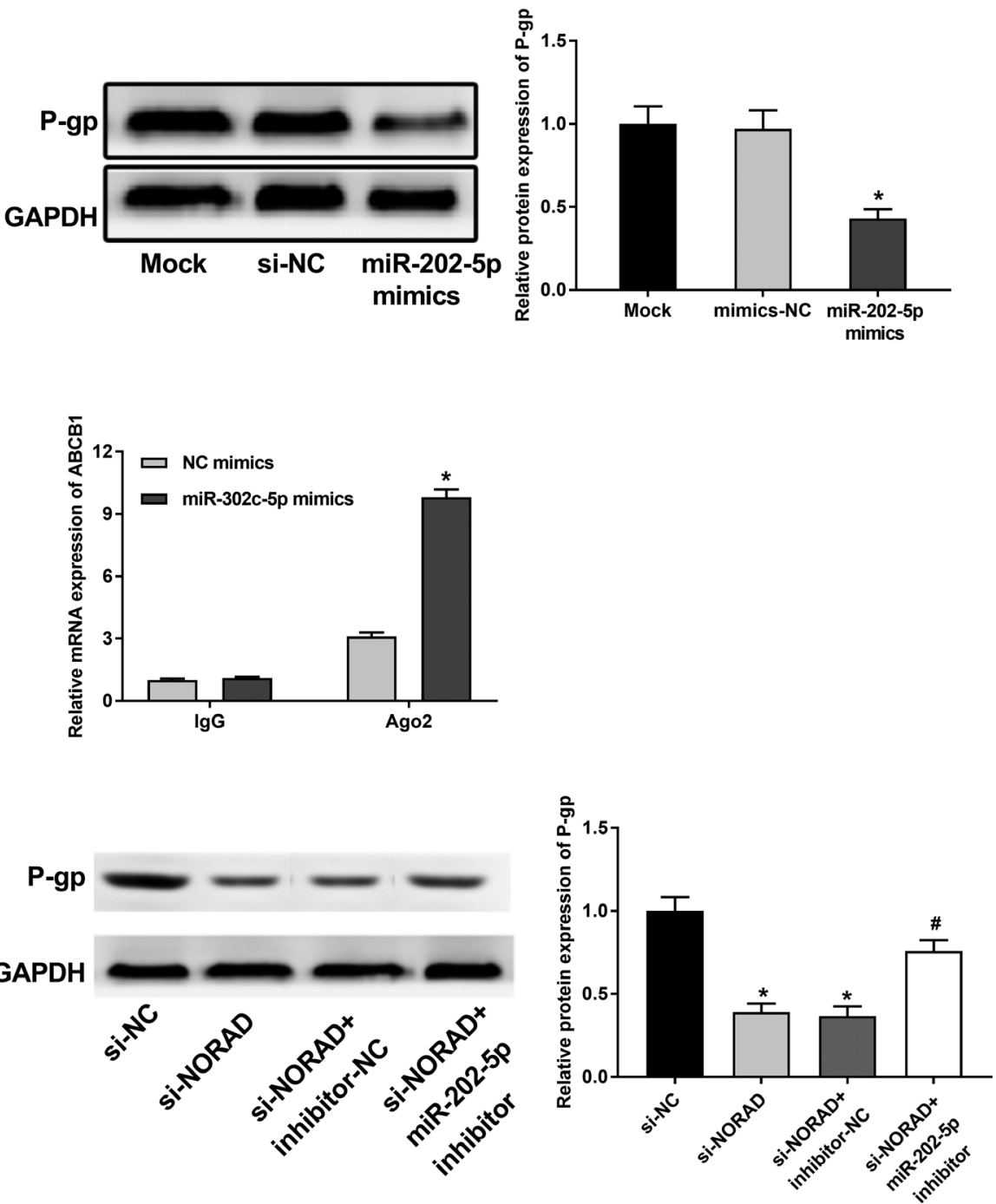

Figure 5. Permeability glycoprotein (P-gp) is the target of NORAD/miR-202-5p. A. The binding sites of P-gp and miR-202-5p were identified by searching TargetScan (http://www.targetscan.org/vert_72/). B. The expression of ABCB1 mRNA and P-gp protein detected by qRT-PCR and Western blot after transfecting miR-202-5p mimics. ${ }^{*} p<0.05$ compared with the mimics negative control (NC) group. C. Validation of miR-202-5p binding to ABCB1 by dual-luciferase activity assay and anti-Ago2 RNA immunoprecipitation assay. D. The expression of ABCB1 mRNA and P-gp protein after the knockdown of NORAD or the combination of knockdown of NORAD and inhibition of miR-202-5p. Data are shown as means \pm SD from three independent experiments. ${ }^{*} p<0.05$ compared with the si-NC group, ${ }^{\#} p<0.05$ compared with the si-NORAD+inhibitor-NC group. 
A

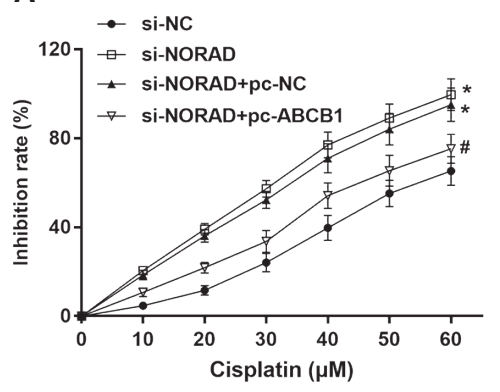

B

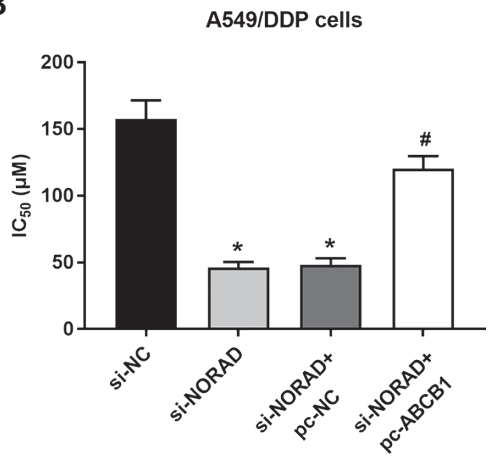

Figure 6. $\mathrm{ABCB} 1$ overexpression eliminates the effect of NORAD silencing in A549/DDP cells. A. The inhibition rate in A549/DDP co-transfected with $A B C B 1$ overexpression (pc-ABCB1) and si-NORAD. B. The $\mathrm{IC}_{50}$ value in A549/DDP co-transfected with pc-ABCB1 and si-NORAD. Data are shown as means \pm SD from three independent experiments. ${ }^{*} p<0.05$ compared with the si-NC group, ${ }^{\#} p<0.05$ compared with the si-NORAD+pc-NC group.
NORAD functions as a promoter in A549/DDP cells' drug resistance. Mechanically, high level of NORAD upregulated P-gp expression by functioning as a competing endogenous RNAs (ceRNA) of miR-202-5p. Thus, we identified a novel NORAD/miR-202-5p/P-gp axis in the acquisition of DDP resistance of A549/DDP cells.

Growing evidence have shown that lncRNAs could sponge the miRNAs as a ceRNA to indirectly regulate the expression of target genes (Tay et al. 2014). It is reported that NORAD may function as a ceRNA to promote metastasis in pancreatic cancer by competing with miR125a-3p (Li et al. 2017). Thus, in the present study, we hypothesised that NORAD could sponge the miRNAs as ceRNAs to exert its influence on DDP resistance development. Bioinformatics analysis revealed that NORAD includes a targeting site of miR-202-5p. Dual-luciferase reporter assay also showed that miR-202-5p could directly bind to NORAD via a putative miRNA response element. NORAD knockdown increased miR-202-5p expression in A549/DDP cells, and inhibition of miR-202-5p also increased NORAD expression. Moreover, the inhibition of miR-202-5p could reverse the effect of NORAD knockdown on DDP resistance. This rescue assay confirmed that NORAD regulated A549/DDP cell chemosensitivity by targeting miR-202-5p.

P-gp was considered to be one of the critical factors for the induction of drug resistance (Kumar et al. 2019). In this study, we further investigated whether NORAD could enhance the drug resistance of A549/DDP cells by regulating P-gp. Our results showed that knocking down NORAD suppresses the expression of P-gp. Moreover, both bioinformatics analysis and dual-luciferase reporter gene assay revealed that miR-202-5p could bind to the 3'-UTR of P-gp gene. Therefore, NORAD regulated the expression of P-gp by modulating the level of miR-202-5p.

In conclusion, we found that NORAD could enhance the drug resistance of A549 to DDP, and its potential mechanism was to adsorb and down-regulate the miR-202-5p expression, promoting P-gp expression. This study may illuminate a new potential mechanism of drug resistance in NSCLC cells.

Acknowledgements. Not applicable.

Conflict of interest. The authors declare that there is no conflict of interest.

\section{References}

Aller S, Yu J, Ward A, Weng Y, Chittaboina S, Zhuo R, Harrell P, Trinh Y, Zhang Q, Urbatsch I, Chang G (2009): Structure of P-glycoprotein reveals a molecular basis for poly-specific drug binding. Science NLM 323, 1718-1722 https://doi.org/10.1126/science.1168750

Arbour KC, Riely GJ (2019): Systemic therapy for locally advanced and metastatic non-small cell lung cancer: A Review. JAMA 322, 764-774 https://doi.org/10.1001/jama.2019.11058

Barton MK (2017): Encouraging long-term outcomes reported in patients with stage I non-small cell lung cancer treated with stereotactic ablative radiotherapy. CA Cancer J. Clin. 67, 349-350 https://doi.org/10.3322/caac.21375

Chen FF, Lv X, Zhao QF, Xu YZ, Song SS, Yu W, Li XJ (2018): Inhibitor of DNA binding 3 reverses cisplatin resistance in human lung adenocarcinoma cells by regulating the PI3K/Akt pathway. Oncol. Lett. 16, 1634-1640 https://doi.org/10.3892/ol.2018.8849

Chian S, Zhao Y, Xu M, Yu X, Ke X, Gao R, Yin L (2019): Ginsenoside Rd reverses cisplatin resistance in non-small-cell lung cancer A549 cells by downregulating the nuclear factor erythroid 2-related factor 2 pathway. Anti-Cancer Drugs 30, 838-845 https://doi.org/10.1097/CAD.0000000000000781

Dong Y, Xu T, Zhong S, Wang B, Zhang H, Wang X, Wang P, Li G, Yang S (2019): Circ_0076305 regulates cisplatin resistance of non-small cell lung cancer via positively modulating STAT3 by sponging miR-296-5p. Life Sci. 239, 21 https://doi.org/10.1016/j.lfs.2019.116984

Hu B, Zhang H, Wang Z, Zhang F, Wei H, Li L (2017): LncRNA CCAT $1 /$ miR-130a-3p axis increases cisplatin resistance in 
non-small-cell lung cancer cell line by targeting SOX4. Cancer Biol. Ther. 18, 974-983 https://doi.org/10.1080/15384047.2017.1385679

Huang Q, Xing S, Peng A, Yu Z (2020): NORAD accelerates chemo-resistance of non-small-cell lung cancer via targeting at miR-129-1-3p/SOX4 axis. Biosci. Rep. 40 https://doi.org/10.1042/BSR20193489

Kumar A, Jaitak V (2019): Natural products as multidrug resistance modulators in cancer. Eur. J. Med. Chem. 176, 268-291 https://doi.org/10.1016/j.ejmech.2019.05.027

Li H, Wang X, Wen C, Huo Z, Wang W, Zhan Q, Cheng D, Chen H, Deng X, Peng C, Shen B (2017): Long noncoding RNA NORAD, a novel competing endogenous RNA, enhances the hypoxia-induced epithelial-mesenchymal transition to promote metastasis in pancreatic cancer. Mol. Cancer 16, 169 https://doi.org/10.1186/s12943-017-0738-0

Li Q, Li C, Chen J, Liu P, Cui Y, Zhou X, Li H, Zu X (2018a): High expression of long noncoding RNA NORAD indicates a poor prognosis and promotes clinical progression and metastasis in bladder cancer. Urologic Oncology 36, 310.e315-310.e322 https://doi.org/10.1016/j.urolonc.2018.02.019

Li Y, Sun J, Gao S, Hu H, Xie P (2018b): HOXB4 knockdown enhances the cytotoxic effect of paclitaxel and cisplatin by downregulating $\mathrm{ABC}$ transporters in ovarian cancer cells. Gene 663, 9-16 https://doi.org/10.1016/j.gene.2018.04.033

Liu H, Li J, Koirala P, Ding X, Chen B, Wang Y, Wang Z, Wang C, Zhang X, Mo YY (2016): Long non-coding RNAs as prognostic markers in human breast cancer. Oncotarget 7, 20584-20596 https://doi.org/10.18632/oncotarget.7828

Lu T, Wang Y, Chen D, Liu J, Jiao W (2018): Potential clinical application of lncRNAs in non-small cell lung cancer. Onco. Targets Ther. 11, 8045-8052 https://doi.org/10.2147/OTT.S178431

Nagasaka M, Gadgeel SM (2018): Role of chemotherapy and targeted therapy in early-stage non-small cell lung cancer. Expert Rev. Anticanc. 18, 63-70 https://doi.org/10.1080/14737140.2018.1409624

Rafiee A, Riazi-Rad F, Havaskary M, Nuri F (2018): Long noncoding RNAs: regulation, function and cancer. Biotechnol. Genet. Eng. 34, 153-180 https://doi.org/10.1080/02648725.2018.1471566
Ramanathan K, Shanthi V, Maiti S, Shin WH, Kihara D (2019): Current progress and future perspectives of polypharmacology: From the view of non-small cell lung cancer. Semin. Cancer Biol. 2019

Schmitt AM, Chang HY (2016): Long noncoding RNAs in cancer pathways. Cancer Cell 29, 452-463 https://doi.org/10.1016/j.ccell.2016.03.010

Tao W, Li Y, Zhu M, Li C, Li P (2019): LncRNA NORAD promotes proliferation and inhibits apoptosis of gastric cancer by regulating miR-214/Akt/mTOR axis. Onco. Targets Ther. 12, $8841-8851$ https://doi.org/10.2147/OTT.S216862

Tay Y, Rinn J, Pandolfi PP (2014): The multilayered complexity of ceRNA crosstalk and competition. Nature 505, 344 https://doi.org/10.1038/nature12986

Tian LJ, Wu YP, Wang D, Zhou ZH, Xue SB, Zhang DY, Wei YG, Liu W (2019): Upregulation of long noncoding RNA (lncRNA) X-inactive specific transcript (XIST) is associated with cisplatin resistance in non-small cell lung cancer (NSCLC) by downregulating microRNA-144-3p. Med. Sci. Monit. 25, 8095-8104 https://doi.org/10.12659/MSM.916075

Tong L, Ao Y, Zhang H, Wang K, Wang Y, Ma Q (2019): Long noncoding RNA NORAD is upregulated in epithelial ovarian cancer and its downregulation suppressed cancer cell functions by competing with miR-155-5p. Cancer Med. 8, 4782-4791 https://doi.org/10.1002/cam4.2350

Wu CP, Hsieh CH, Wu YS (2011): The emergence of drug transporter-mediated multidrug resistance to cancer chemotherapy. Mol. Pharmaceut. 8, 1996-2011 https://doi.org/10.1021/mp200261n

Zhang H, Guo H (2019): Long non-coding RNA NORAD induces cell proliferation and migration in prostate cancer. J. Int. Med. Res. 47, 3898-3904 https://doi.org/10.1177/0300060519862076

Zhang J, Li XY, Hu P, Ding YS (2018): LncRNA NORAD contributes to colorectal cancer progression by inhibition of miR-202-5p. Oncol. Res. 26 https://doi.org/10.3727/096504018X15190844870055

Received: February 19, 2020

Final version accepted: July 12, 2020 